\title{
BMJ Open Treatment pathways in people with type 2 diabetes mellitus: a nationwide cohort study of new users of metformin monotherapy in New Zealand
}

\author{
Joyce Guo, ${ }^{1}$ Lianne Parkin (D) , ${ }^{2,3}$ Jiaxu Zeng, ${ }^{2,3}$ David Barson, ${ }^{2,3}$ \\ Simon Horsburgh ${ }^{2,3}$
}

To cite: Guo J, Parkin L, Zeng J, et al. Treatment pathways in people with type 2 diabetes mellitus: a nationwide cohort study of new users of metformin monotherapy in New Zealand. BMJ Open 2021;11:e051884. doi:10.1136/ bmjopen-2021-051884

\section{- Prepublication history and} additional supplemental material for this paper are available online. To view these files, please visit the journal online. (http://dx.doi.org/10.1136/ bmjopen-2021-051884).

$J G$ and $L P$ are joint first authors.

Received 31 March 2021 Accepted 15 July 2021

Check for updates

(c) Author(s) (or their employer(s)) 2021. Re-use permitted under CC BY-NC. No commercial re-use. See rights and permissions. Published by BMJ.

${ }^{1}$ Medical Student, Dunedin School of Medicine, University of Otago, Dunedin, New Zealand

${ }^{2}$ Pharmacoepidemiology Research Network, University of Otago, Dunedin, New Zealand

${ }^{3}$ Department of Preventive and Social Medicine, Dunedin School of Medicine, University of Otago, Dunedin, New Zealand

Correspondence to

Lianne Parkin;

lianne.parkin@otago.ac.nz

\section{ABSTRACT}

Objectives The aims of this study were to describe the following: (1) the time to change of therapy in patients with type 2 diabetes who had initiated metformin monotherapy as first-line treatment and (2) the sequence in which subsequent therapeutic regimens were introduced. Design Cohort study.

Setting National study based on linked data from the New Zealand Ministry of Health's National Collections of health and pharmaceutical dispensing data.

Participants People with type 2 diabetes mellitus who initiated metformin monotherapy between 1 January 2006 and 30 September 2014 ( $n=93874)$.

Primary outcome measures Cumulative incidence curves were plotted to show the time taken to move from one regimen to another, while sunburst plots were used to illustrate the sequence in which regimens were introduced.

Results About $10 \%$ and $35 \%$ of cohort members had moved to a second regimen 1 year and 5 years, respectively, after initiating metformin monotherapy; the majority received a regimen recommended by New Zealand treatment guidelines (mostly metformin and a sulphonylurea). Of those who started a recommended second regimen, $37 \%$ and $67 \%$ had moved to a third regimen after 1 and 5 years, respectively; the corresponding proportions for those who started an 'other' (not listed as recommended) second regimen were $53 \%$ and $75 \%$. Most of those who received a third regimen after a recommended second regimen were dispensed an 'other' third regimen. Of those who moved to a third regimen from an 'other' second regimen, similar proportions received recommended and 'other' third regimens.

Conclusions Real-world type 2 diabetes treatment patterns in New Zealand are complex and not always consistent with guidelines.

\section{INTRODUCTION}

Type 2 diabetes mellitus is a major public health issue in New Zealand. Data from the 2018/2019 New Zealand Health Survey (NZHS) suggest that about $6.4 \%$ of the overall New Zealand population aged $\geq 25$ years has type 2 diabetes, with even higher proportions
Strengths and limitations of this study

- This nationwide study in New Zealand explored realworld treatment pathways among people with type 2 diabetes who had initiated pharmacological treatment with metformin monotherapy.

- The study was based on a national pharmaceutical claims database that covers the entire population of New Zealand.

- It was not possible to examine the use of antidiabetic drugs which were approved for use but not publicly funded during the study period; however, the proportionate use of these drugs would have been very small.

- Lack of access to detailed clinical data meant it was not possible to explore the appropriateness of changes in treatment, or detect situations in which treatment should have been escalated but was not, or to identify changes in therapy that occurred because of intolerance to metformin.

for Māori (the indigenous people of New Zealand), Pacific and Asian populations. ${ }^{1}$ Moreover, the prevalence is increasing over time-according to data from the Virtual Diabetes Register (VDR), the number of people with diabetes (most of whom will have type 2 diabetes) rose progressively between 2010 and $2018 .^{2}$ The true prevalence of type 2 diabetes is likely to be even higher than suggested by the NZHS and VDR estimates as they are based on diagnosed diabetes only.

Good glycaemic control is fundamental to preventing diabetes-related complications and type 2 diabetes management guidelines published by the New Zealand Guidelines Group in June 2011 recommended that treatment is tailored to maintain glycated haemoglobin $\left(\mathrm{HbA}_{\mathrm{c}}\right)<50-55 \mathrm{mmol} / \mathrm{mol}$, or some other target agreed with the patient. ${ }^{3}$ The guidelines advocated a 3-step approach to pharmacological treatment. First, metformin monotherapy should be initiated 
as the first-line treatment and if this is not tolerated or is contraindicated, sulphonylurea monotherapy should be prescribed instead. Alternatively, acarbose could be used, if tolerated. Second, if a patient's $\mathrm{HbA1}{ }_{c}$ remains above the agreed target after 3 months, despite optimal dosage and adherence to metformin, a sulphonylurea should be added. If metformin and a suphonylurea are not tolerated or are contraindicated, or an alternative to insulin is required, then pioglitazone monotherapy could be prescribed, or a dipeptidyl peptidase-4 (DPPIV) inhibitor. Third, if glycaemic control remains poor after 3 months, despite optimal dosage and adherence to second-line therapy, insulin should be added; isophane insulin is the first choice, but in certain circumstances basal insulin analogues or pre-mixed insulin preparations might be more appropriate. Alternatively, a DPP-IV inhibitor or a glucagon-like peptide-1 (GLP-1) agonist could be used.

The New Zealand Guidelines Group was disestablished in 2012, however subsequent guidelines and consensus statements from the New Zealand Best Practice Advocacy Centre, ${ }^{4}$ the UK National Institute for Health and Care Excellence, ${ }^{5}$ the Scottish Intercollegiate Guidelines Network, ${ }^{6}$ the European Association for the Study of Diabetes $^{7}$ and the American Diabetes Association ${ }^{7}$ have all recommended a stepwise intensification from metformin monotherapy to dual therapy with oral hypoglycaemic agents, followed by triple therapy with oral agents or the addition of insulin.

The treatment pathways in patients with type 2 diabetes have not been fully explored in New Zealand. One study examined the use of antidiabetic agents in people who commenced drug treatment for type 2 diabetes during a 9-year period (financial years 2007/2008-2015/2016). ${ }^{8}$ The majority of patients initiated metformin monotherapy (increased from $80 \%$ to $85 \%$ over the study period), while small proportions initiated metformin with insulin (increased from 2\% to $5 \%$ ), metformin with a sulphonylurea (decreased from $10 \%$ to $6 \%$ ), sulphonylurea-monotherapy (decreased from $8 \%$ to $2 \%$ ) and other therapy (stable at 1\%). The researchers undertook some limited follow-up of patients who commenced drug treatment in $2007 / 2008$, but concluded there was a need for further research, including an exploration of the time to escalation of therapy.

We undertook a national study to describe the treatment pathways in an existing cohort of patients who initiated metformin monotherapy for type 2 diabetes in New Zealand between 2006 and 2014. The specific aims of the study were as follows:

1. To describe the time to change in therapy from:

i. Metformin monotherapy to a second therapeutic regimen (overall, recommended, 'other').

ii. Recommended second therapeutic regimen to a third therapeutic regimen (overall, recommended, 'other'). iii. 'Other' second therapeutic regimen to a third therapeutic regimen (overall, recommended, 'other').

2. To repeat the above analyses for the periods before, and after, the publication of type 2 diabetes management guidelines by the New Zealand Guidelines Group in 2011.

3. To describe the sequence in which unique therapeutic regimens were subsequently introduced in patients who initiated metformin monotherapy.

\section{METHODS}

\section{Data sources and identification of the study cohort}

New Zealand has a universal healthcare system in which hospital care and subsidised prescription medicines are provided to all citizens and permanent residents (about 5 million people). ${ }^{9}$ Summary data regarding pharmaceutical dispensings, hospital discharges, deaths and demographic characteristics are held centrally in the Ministry of Health's National Collections. ${ }^{10}$

This study is based on an existing national cohort of people who initiated metformin monotherapy for type 2 diabetes between 1 January 2006 and 30 September 2014. The methods used to derive the original cohort have been described in detail elsewhere ${ }^{11}$ but, in brief, the Ministry of Health identified all people listed on the VDR $^{2}$ between 1 January 2005 and 31 December 2014, including those who died and those who were not registered with a Primary Health Organisation during any given year. For each of these individuals, the Ministry provided us with the following data from the National Collections, using an encrypted unique patient identifier (the National Health Index (NHI)) as the linkage key: demographic information (from the NHI Collection), details of all publicly funded dispensings of prescription medicines between 1 January 2005 and 31 December 2015 (from the Pharmaceutical Collection), details of any publicly funded hospital discharges between 1 January 1988 and 31 December 2015 (from the National Minimum Dataset), and for those who died between cohort entry (first metformin monotherapy dispensing) and 31 December 2015, the date and causes of death (from the Mortality Collection). We then used these data to identify the cohort for this study, as outlined in online supplemental efigure 1.

\section{Identifying therapeutic regimens used by members of the study cohort}

For each member of the study cohort, we summarised exposure to oral hypoglycaemic agents and insulin during follow-up employing a similar approach to one we have used previously. ${ }^{12}$ This involved several steps. First, we combined the dispensing data relating to each of the relevant drugs (online supplemental etable) into continuous episodes of use; a continuous episode was defined as a series of dispensings in which the elapsed time between the end date of one dispensed supply and the start date 
of the next was $\leq 30$ days (online supplemental efigure 2A). We calculated the end date of a dispensed supply by adding the recorded number of days supplied to the date of dispensing; if the days' supply was not recorded in the data the Ministry provided, we set the end date to the earlier of the date of the next dispensing minus 1 day or the date of dispensing plus 90 days (as this is the maximum supply that can be legally provided on one prescription). Second, we summarised the continuous episodes of use of individual drugs into continuous episodes of use of drug classes (online supplemental efigure 2B). Third, we aggregated the data into continuous episodes of use of mutually exclusive therapeutic regimens (online supplemental efigure 3). Follow-up was censored at the earlier of the date of death or 31 December 2015 (end of the study period).

The second and third therapeutic regimens used by individuals, if any, were classified as 'recommended' in accordance with the type 2 diabetes management guidelines published by the New Zealand Guidelines Group in 2011. ${ }^{3}$ Therefore, recommended second-line therapy was defined as the use of metformin plus a sulphonylurea, or the use of pioglitazone monotherapy, following the use of metformin monotherapy. Any other second therapeutic regimen was classified as 'other'. Recommended thirdline therapy was defined as the use, following a second therapeutic regimen (whether recommended or not), of insulin (isophane insulin, basal insulin analogues, or premixed insulin preparations) with or without oral hypoglycaemic agents or short-acting insulin. Any other third therapeutic regimen was classified as 'other'.

\section{Statistical methods}

Cumulative incidence curves were plotted to summarise the time taken to move from (1) metformin monotherapy to a second therapeutic regimen (overall, recommended, 'other'), (2) a recommended second therapeutic regimen to a third therapeutic regimen (overall, recommended, 'other') and (3) an 'other' second therapeutic regimen to a third therapeutic regimen (overall, recommended, 'other'). Death was treated as a competing event for all analyses. To estimate the cumulative incidence of change to a recommended regimen, change to an 'other' regimen was considered an additional competing event (and vice versa). All individuals who entered the cohort between 1 January 2006 and 30 September 2014 were included in these analyses. Separate cumulative incidence curves were produced for people who entered the cohort before 1 January 2011 and after 31 December 2011 (preguideline and post-guideline groups, respectively).

Sunburst plots were created to illustrate the order in which unique therapeutic regimens were used by cohort members in the first year, first 2 years and first 3 years after cohort entry (among those with $\geq 1$ year, $>2$ years and $\geq 3$ years of follow-up, respectively) using methods described by others ${ }^{13}$ and previously employed by our group. ${ }^{12}$ Plots were generated for people who entered the cohort at any time between 1 January 2006 and 30 September
2014 and had the required amount of follow-up, as well as those who entered pre-guidelines and post-guidelines. To ensure a clear delineation between prescribing practices before and after the guidelines were published in June 2011, we restricted the pre-guideline analyses to individuals who had completed the requisite years of follow-up (1, 2 or 3 years) before 1 January 2011 and restricted the post-guideline analyses to those who completed the requisite years of follow-up between 1 January 2012 and 31 December 2015.

All dataset manipulations were performed using SAS V.9.4. ${ }^{14}$ Sunburst plots and cumulative incidence curves were plotted in R V.3.5.1. ${ }^{15}$

\section{Patient and public involvement}

The study was based on anonymised routinely collected data and patients were not involved.

\section{RESULTS}

A total of 93874 individuals initiated metformin monotherapy for type 2 diabetes between 1 January 2006 and 30 September 2014 (online supplemental efigure 1). The characteristics of these individuals at cohort entry are shown in table 1 .

Figures 1-3 show the proportions of cohort members who moved, over time, from metformin monotherapy to a second therapeutic regimen, from a recommended second therapeutic regimen to a third therapeutic regimen, and from an 'other' second therapeutic regimen to a third therapeutic regimen. About $10 \%$ and $35 \%$ of cohort members had moved to a second regimen 1 year and 5 years, respectively, after initiating metformin monotherapy; the majority received a recommended regimen (figure 1). Of those who had moved to a recommended second regimen, about $37 \%$ and $67 \%$ had started a third regimen 1 year and 5 years after treatment intensification (figure 2); the corresponding figures for those who had moved to an 'other' second regimen were 53\% and $75 \%$ (figure 3). Most of those who received a third regimen after a recommended second regimen were dispensed an 'other', regimen, whereas the proportionate use of recommended and 'other' third regimens was similar among those who moved from an 'other' second regimen. The analogous cumulative incidence curves for individuals who entered the cohort before and after 2011 are shown in online supplemental efigures 4-9. In general, the findings for those who entered the cohort in the pre-guideline and post-guideline years were similar, except for individuals who moved from an 'other' second regimen to a third regimen; in the pre-guideline years higher proportions used an 'other' third regimen, but post-guidelines higher proportions used a recommended third regimen.

Figure 4 shows the proportions and sequence of use of unique therapeutic regimens in the first year, first 2 years and first 3 years following initiation of metformin monotherapy, counting only the first exposure to any 


\begin{tabular}{|c|c|}
\hline Characteristic & $\begin{array}{l}\text { Number }(\%) \\
(n=93874)\end{array}$ \\
\hline \multicolumn{2}{|l|}{ Sex } \\
\hline Female & $45056(48.0)$ \\
\hline Male & $48818(52.0)$ \\
\hline \multicolumn{2}{|l|}{ Age group (years) } \\
\hline$<25$ & $1349(1.4)$ \\
\hline $25-34$ & $4510(4.8)$ \\
\hline $35-44$ & $12004(12.8)$ \\
\hline $45-54$ & $22800(24.3)$ \\
\hline $55-64$ & $24889(26.5)$ \\
\hline $65-74$ & $18262(19.5)$ \\
\hline$\geq 75$ & $10060(10.7)$ \\
\hline \multicolumn{2}{|l|}{ Ethnicity (prioritised) ${ }^{\star}$} \\
\hline Māori & $14884(15.9)$ \\
\hline Pacific & $12337(13.1)$ \\
\hline European & $49191(52.4)$ \\
\hline Asian (non-Indian) & $6727(7.2)$ \\
\hline Indian & $6098(6.5)$ \\
\hline Middle Eastern/Latin American/African & $1150(1.2)$ \\
\hline Other & $188(0.2)$ \\
\hline Unknown & $3299(3.5)$ \\
\hline \multicolumn{2}{|c|}{ Socioeconomic deprivation (NZDep2013 decile)† } \\
\hline 1 (least deprived) & $5619(6.0)$ \\
\hline 2 & $6263(6.7)$ \\
\hline 3 & $6607(7.0)$ \\
\hline 4 & $7321(7.8)$ \\
\hline 5 & $8016(8.5)$ \\
\hline 6 & $8318(8.9)$ \\
\hline 7 & 10469 (11.2) \\
\hline 8 & 11049 (11.8) \\
\hline 9 & $13820(14.7)$ \\
\hline 10 (most deprived) & $16199(17.3)$ \\
\hline Unknown & $193(0.2)$ \\
\hline
\end{tabular}

*Self-identified ethnicity categorised according to the Ministry of Health Ethnicity Data Protocols. ${ }^{26}$

†New Zealand Deprivation Index, an area-based measure of social deprivation. $^{27}$

given regimen across the full study period. Of the cohort members who moved from metformin monotherapy to a second therapeutic regimen in the first year of follow-up, about $72 \%$ used metformin plus a sulphonylurea and a very small proportion used pioglitazone monotherapy (the recommended second-line therapies), while $14 \%$ used sulphonylurea monotherapy, $10 \%$ used insulincontaining regimens and smaller proportions used various other regimens. Of those who moved to a third regimen in the first year of follow-up, the majority who

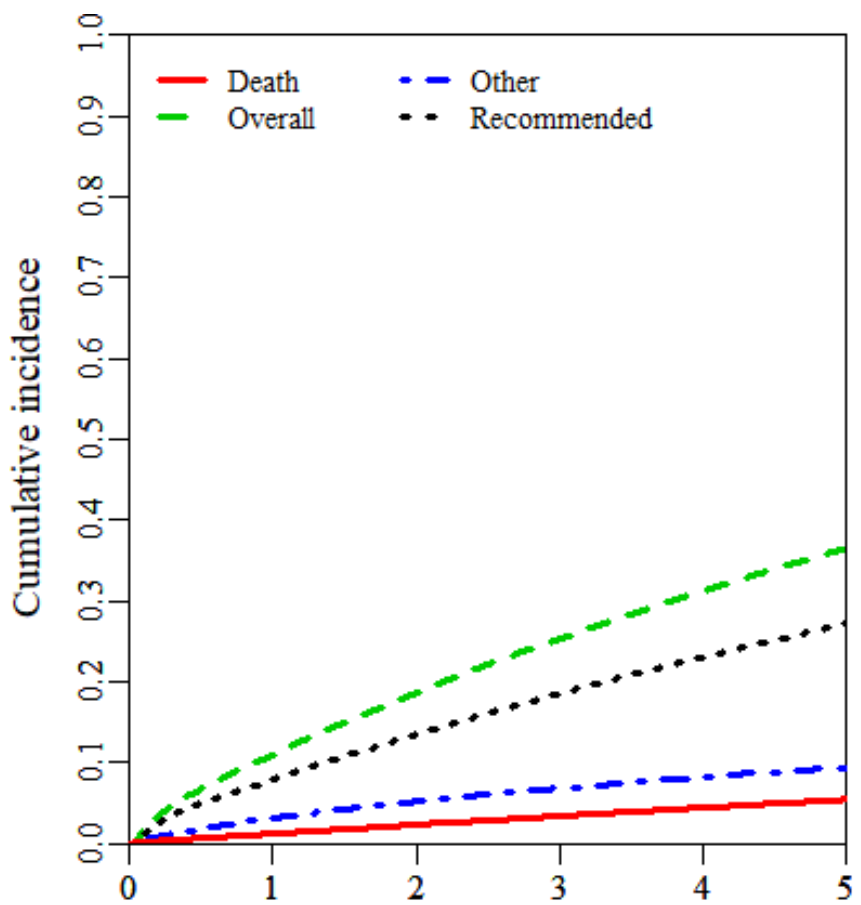

Time since initiation of metformin monotherapy (years)

Figure 1 Time taken from initiation of metformin monotherapy to change to a second therapeutic regimen.

had used metformin plus a sulphonylurea as a second regimen moved to sulphonylurea monotherapy, while the majority of those on metformin plus insulin moved to

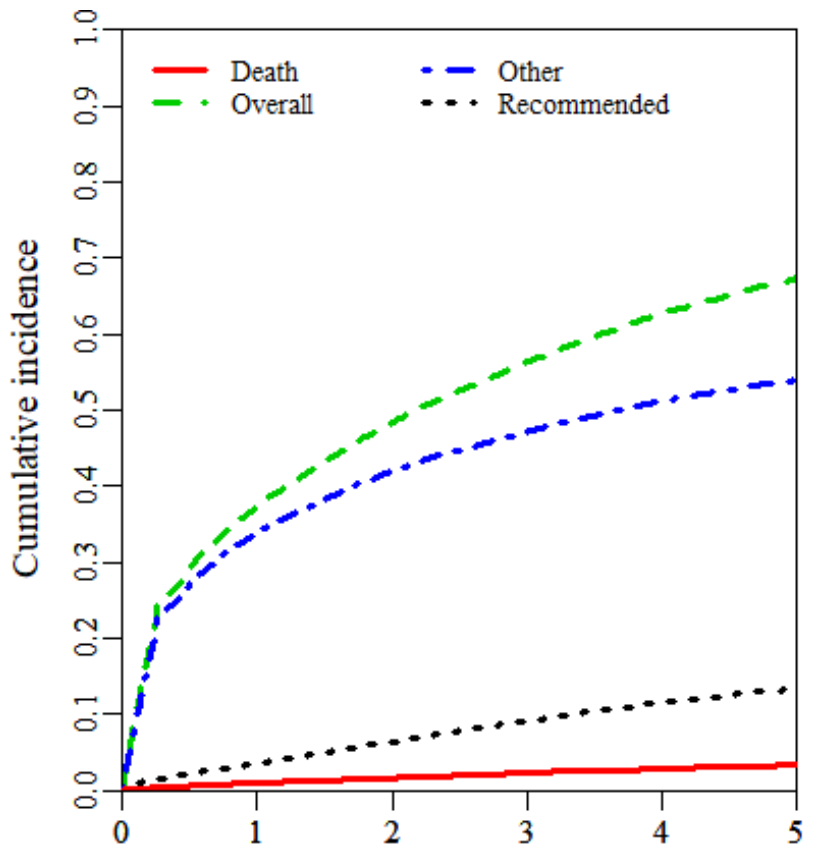

Time since initiation of recommended second regimen (years)

Figure 2 Time taken from initiation of a recommended second therapeutic regimen to change to a third therapeutic regimen. 


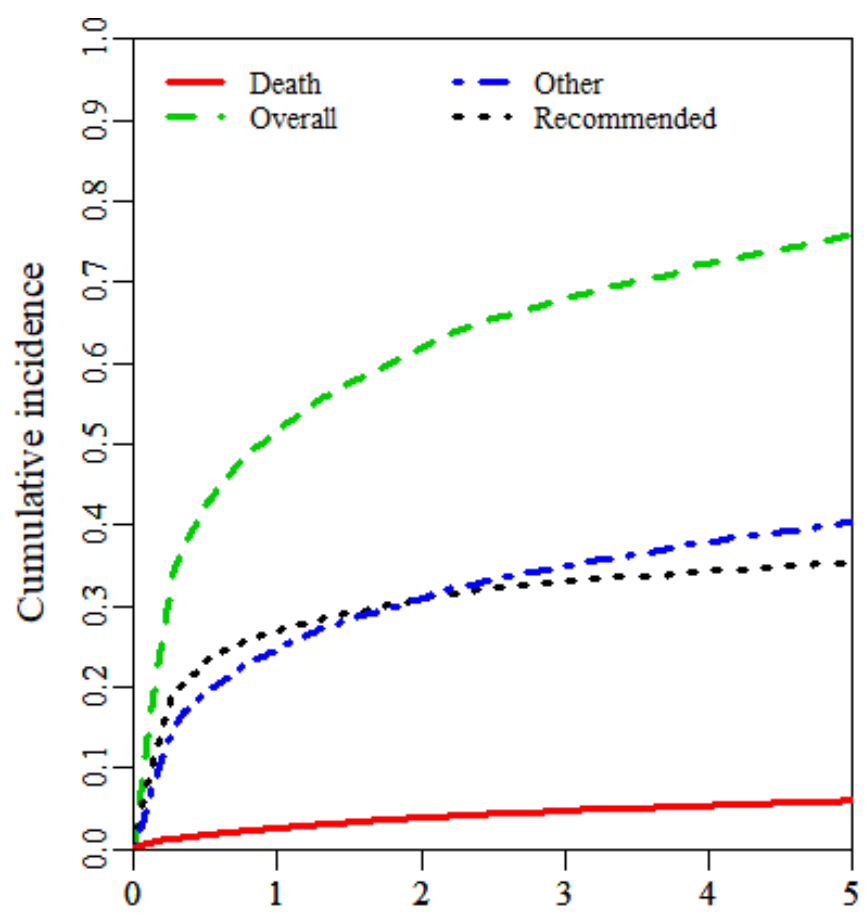

Time since initiation of "other" second regimen (years)

Figure 3 Time taken from initiation of an 'other' second therapeutic regimen to change to a third therapeutic regimen.

insulin alone, and the majority on sulphonylurea monotherapy moved to metformin plus a sulphonylurea. The proportions of cohort members who moved to second and third regimens increased with longer follow-up times, but the general patterns of regimen use remained the same. The corresponding sunburst plots for the preguideline and post-guideline periods are shown in online supplemental efigures 10 and 11 .
We also undertook sensitivity analyses to investigate whether the high proportion of 'other' third therapeutic regimens among those who had received a recommended second regimen was likely to have been an artefact of how the regimens were constructed-for example, cohort members who appeared to have moved from metformin monotherapy to metformin plus sulphonylurea to sulphonylurea monotherapy might have been instructed by their doctors to switch from metformin monotherapy to sulphonylurea monotherapy, rather than to add the sulphonylurea to metformin. We therefore explored the impact on the cumulative incidence curves of excluding second and any subsequent therapeutic regimens with a duration of $<15$ days, $<30$ days and $<60$ days. While this slightly decreased the proportions moving to second and third regimens, the overall patterns in relation to the use of recommended and 'other' regimens were very similar to the cumulative incidence curves in the main analyses.

\section{DISCUSSION}

In this national cohort study of people who initiated metformin monotherapy for type 2 diabetes during a 9-year period, we found that relatively small proportions of cohort members moved to a second therapeutic regimen in the first few years of follow-up. The majority of these people received a second-line regimen recommended by contemporaneous New Zealand treatment guidelines (almost all metformin plus a sulphonylurea). Although most of those who moved to a third regimen from a recommended second regimen received an 'other' regimen, there was evidence of improvement over time, with a higher proportion transitioning to a recommended third regimen.

This study has several strengths. It is the first nationwide study in New Zealand to have undertaken an in-depth

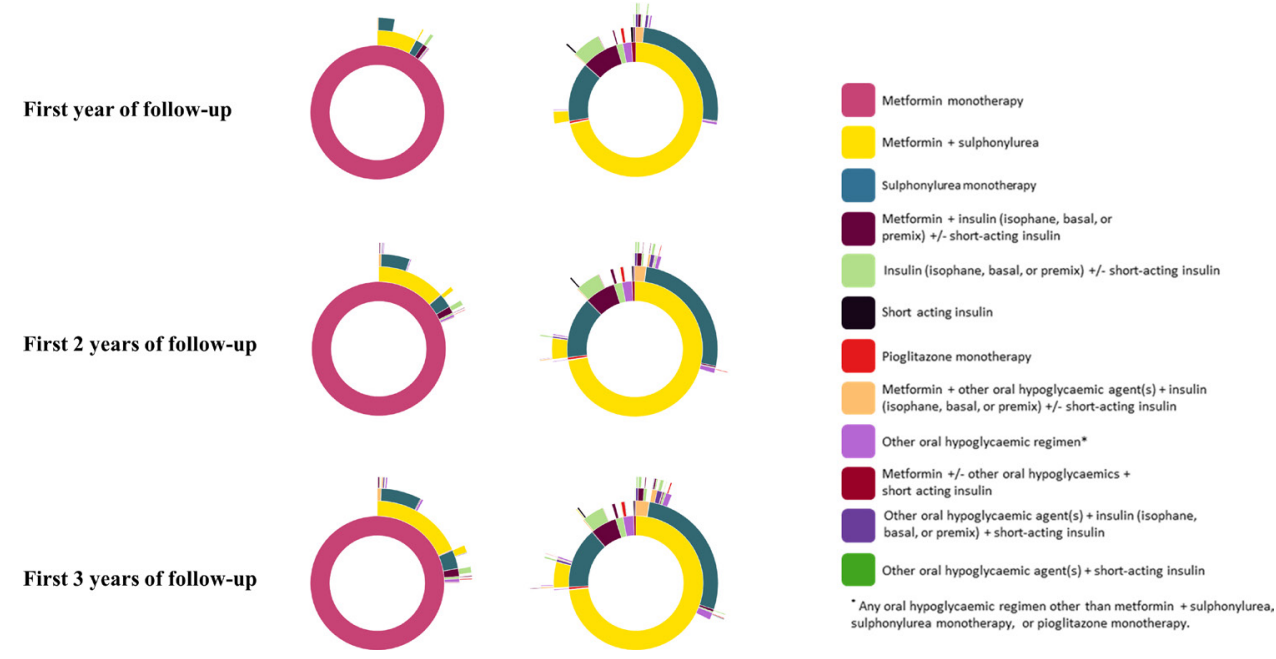

Figure 4 Proportions and sequence of use of unique therapeutic regimens in the first year, first 2 years and first 3 years following initiation of metformin monotherapy. The sunburst plots on the left illustrate the proportions of metformin monotherapy users (inner ring) who moved to a second regimen (second ring), the proportions who moved to a third regimen (third ring) according to the second regimen they had used, and so on, in the first year, first 2 years and first 3 years of follow-up. The plots on the right show, in more detail, the second regimens that were used (inner ring), as well as the subsequent regimens. 
exploration of treatment pathways in people with type 2 diabetes over an extended period. The use of the Pharmaceutical Collection as a source of information about diabetes treatments is a further strength-the database contains records of subsidised dispensings of medicines for the entire population of New Zealand and it is likely that these records are virtually complete since pharmacists are not remunerated for such dispensings unless they submit a reimbursement claim. Although the Pharmaceutical Collection does not include medicines dispensed in hospital, it does include medicines prescribed at discharge as patients collect these from community pharmacies.

The study also has some limitations. First, we were unable to examine the use of antidiabetic drugs which were approved for use but not publicly funded during the study period as they were not included in the Pharmaceutical Collection. However, while three DPP-IV inhibitors (saxagliptin, sitagliptin and vildagliptin), a GLP-1 agonist (exenatide), and a sodium-glucose co-transporter 2 (SGLT2) inhibitor (dapagliflozin) were approved but unfunded, ${ }^{16}$ we expect that the proportionate use of these drugs would have been very small because of the associated financial burden for patients (personally paying the full costs of the medicines). Second, we did not have access to the results of $\mathrm{HbA} 1_{c}$ tests and therefore were unable to explore the appropriateness of any change in treatment, or detect situations in which treatment should have been escalated but was notthe so-called therapeutic inertia which is increasingly causing concern internationally. ${ }^{17}$ Third, we did not have access to detailed clinical records so we were unable to determine whether people who received sulphonylurea monotherapy as a second regimen did so because of intolerance to metformin. The lack of clinical information also meant that we were solely reliant on the dispensing information to identify the therapeutic regimens used by cohort members. However, the sensitivity analyses suggest that our findings were unlikely to be an artefact of how we constructed the regimens. Fourth, we did not explore the impact of patient characteristics on treatment patterns, as the aim of the study was to provide a simple descriptive overview of treatment pathways in people who initiated metformin monotherapy as first-line treatment for type 2 diabetes. However, this could be investigated in future research. Fifth, we focused on the first exposure to unique regimens and did not look at usage patterns which included switching backwards and forwards between regimens. Future research could use sequence index plots to look at this in further detail. Finally, as this analysis did not include information about doses of medications, we are unable to comment on the possible relations between dosage and changes in treatment.

Internationally, several population-based studies have described the medications prescribed initially, and subsequently, for patients with type 2 diabetes. ${ }^{13}{ }^{18-25}$ Some of these investigations focused on the use of individual drugs, ${ }^{13}{ }^{18-20}$ while others, like this study, examined the use of regimens. ${ }^{21-25}$ However, only some of the studies in the second group examined subsequent regimens according to the first regimen received. ${ }^{21-24}$ Consistent with our findings, the most common second regimen for patients who initiated treatment with metformin monotherapy in the Netherlands, ${ }^{21}{ }^{24}$ the UK, ${ }^{23}{ }^{24}$ Spain ${ }^{24}$ and Ireland ${ }^{22}$ was metformin plus a sulphonylurea, although the proportions were lower than in our study. Conversely, different patterns were observed in France ${ }^{24}$ and Italy. ${ }^{24}$ However, direct comparisons between previous research and our study should be interpreted with caution because different calendar periods were examined and treatment guidelines regarding second-line and third-line therapy differed over time and between countries, as did the availability of new oral hypoglycaemic agents.

In conclusion, this study has provided a useful overview of how type 2 diabetes is being treated in New Zealand. It shows that real-world treatment patterns are complex and not always consistent with guidelines. One DPP-IV inhibitor (vildagliptin) and one SGLT2 inhibitor (empagliflozin) have recently been funded (in October 2018 and February 2021, respectively) and the methods employed in this project could be used in the future to ascertain whether the introduction of these drugs has an impact on treatment pathways in New Zealand.

Correction notice Joint authorship details has been added. "JG and LP are joint first authors."

Acknowledgements We thank Analytical Services at the Ministry of Health for providing the data for this study.

Contributors JG contributed to the design of the study, carried out the analyses, interpreted the findings and contributed to critical revision of the manuscript. LP conceived and designed the study, acquired the data, interpreted the findings and wrote the first draft of the manuscript. JZ provided statistical expertise and contributed to the design of the study, the interpretation of the findings and critical revision of the manuscript. DB provided data management expertise and programmed the study algorithm, derived the analysis dataset and contributed to critical revision of the manuscript. SH provided epidemiological expertise and contributed to the design of the study, the interpretation of the findings and critical revision of the manuscript.

Funding JG received an Otago Medical Research Foundation Summer Scholarship Award to undertake this work.

Disclaimer The funder had no role in the design of the study, or in the collection, analysis, or interpretation of the data, or in the decision to publish.

\section{Competing interests None declared.}

Patient consent for publication Not required.

Ethics approval The study was conducted in accordance with the Declaration of Helsinki and received ethical approval from the University of Otago Human Ethics Committee (Health), reference number HD17/027.

Provenance and peer review Not commissioned; externally peer reviewed.

Data availability statement The data underlying the results presented in the study cannot be shared by the authors because of restrictions imposed by the New Zealand Ministry of Health. Researchers who meet the criteria for access to confidential data can make enquiries with the New Zealand Ministry of Health ( data-enquiries@health.govt.nz).

Supplemental material This content has been supplied by the author(s). It has not been vetted by BMJ Publishing Group Limited (BMJ) and may not have been peer-reviewed. Any opinions or recommendations discussed are solely those of the author(s) and are not endorsed by BMJ. BMJ disclaims all liability and responsibility arising from any reliance placed on the content. Where the content includes any translated material, BMJ does not warrant the accuracy and reliability of the translations (including but not limited to local regulations, clinical guidelines, terminology, drug names and drug dosages), and is not responsible 
for any error and/or omissions arising from translation and adaptation or otherwise.

Open access This is an open access article distributed in accordance with the Creative Commons Attribution Non Commercial (CC BY-NC 4.0) license, which permits others to distribute, remix, adapt, build upon this work non-commercially, and license their derivative works on different terms, provided the original work is properly cited, appropriate credit is given, any changes made indicated, and the use is non-commercial. See: http://creativecommons.org/licenses/by-nc/4.0/.

ORCID iD

Lianne Parkin http://orcid.org/0000-0002-6824-6176

\section{REFERENCES}

1 Ministry of Health. New Zealand Health Survey. Annual Data Explorer. Wellington: Ministry of Health, 2019. https://minhealthnz.shinyapps. io/nz-health-survey-2018-19-annual-data-explorer/_w_9ecba27c/\#!/ home

2 Ministry of Health. Virtual Diabetes Register. Wellington: Ministry of Health, 2019. www.health.govt.nz/our-work/diseases-andconditions/diabetes/about-diabetes/virtual-diabetes-register-vdr

3 New Zealand Guidelines Group. Guidance on the management of type 2 diabetes. Wellington: New Zealand Guidelines Group, 2011.

4 bpac $^{\mathrm{nz}}$. Managing patients with type 2 diabetes: from lifestyle to insulin. Best Pract J 2015;72:32-42 https://bpac.org.nz/BPJ/2015/ December/diabetes.aspx

5 National Institute for Health and Care Excellence. Type 2 diabetes in adults: management. NICE guideline [NG28]. (Updated 2019). London: NICE, 2015. www.nice.org.uk/guidance/ng28/

6 Scottish Intercollegiate Guidelines Network. SIGN 154. Pharmacological management of glycaemic control in people with type 2 diabetes. A national clinical guideline. Edinburgh: Healthcare Improvement Scotland, 2017. www.sign.ac.uk/our-guidelines/ pharmacological-management-of-glycaemic-control-in-people-withtype-2-diabetes/

7 Davies MJ, D'Alessio DA, Fradkin J, et al. Management of hyperglycaemia in type 2 diabetes, 2018. A consensus report by the American Diabetes Association (ADA) and the European Association for the Study of Diabetes (EASD). Diabetologia 2018;61:2461-98.

8 Murray P, Norris H, Metcalfe S, et al. Dispensing patterns for antidiabetic agents in New Zealand: are the guidelines being followed? N Z Med J 2017;130:12-18.

9 Statistics New Zealand. National population estimates: at 30 June 2020. Wellington: Statistics New Zealand, 2020. www.stats.govt.nz/ information-releases/national-population-estimates-at-30-june-2020

10 Ministry of Health Collections. Available: www.health.govt.nz/nzhealth-statistics/national-collections-and-surveys/collections
11 Horsburgh S, Barson D, Zeng J, et al. Adherence to metformin monotherapy in people with type 2 diabetes mellitus in New Zealand. Diabetes Res Clin Pract 2019;158:107902.

12 Parkin L, Barson D, Zeng J, et al. Patterns of use of long-acting bronchodilators in patients with COPD: a nationwide follow-up study of new users in New Zealand. Respirology 2018;23:583-92.

13 Hripcsak G, Ryan PB, Duke JD, et al. Characterizing treatment pathways at scale using the OHDSI network. Proc Natl Acad Sci U S A 2016;113:7329-36.

14 SAS software, version 9.4. SAS Institute Inc., Cary, NC, USA.

15 R Core Team. R: a language and environment for statistical computing. Vienna, Austria: R Foundation for Statistical Computing, 2018. www.r-project.org

16 New Zealand Formulary. Version 73, 2018. Available: https://nzf.org. $\mathrm{nz} /$

17 Khunti K, Gomes MB, Pocock S, et al. Therapeutic inertia in the treatment of hyperglycaemia in patients with type 2 diabetes: a systematic review. Diabetes Obes Metab 2018;20:427-37.

18 Datta-Nemdharry P, Thomson A, Beynon J, et al. Patterns of antidiabetic medication use in patients with type 2 diabetes mellitus in England and Wales. Pharmacoepidemiol Drug Saf 2017;26:127-35.

19 Dennis JM, Henley WE, McGovern AP, et al. Time trends in prescribing of type 2 diabetes drugs, glycaemic response and risk factors: a retrospective analysis of primary care data, 2010-2017. Diabetes Obes Metab 2019;21:1576-84.

20 Curtis HJ, Dennis JM, Shields BM, et al. Time trends and geographical variation in prescribing of drugs for diabetes in England from 1998 to 2017. Diabetes Obes Metab 2018;20:2159-68.

21 Lamberts EJF, Nijpels G, Welschen LMC, et al. Long term patterns of use after initiation of oral antidiabetic drug therapy. Pharmacoepidemiol Drug Saf 2011;20:351-8.

22 Grimes RT, Bennett K, Tilson L, et al. Initial therapy, persistence and regimen change in a cohort of newly treated type 2 diabetes patients. Br J Clin Pharmacol 2015;79:1000-9.

23 Sharma M, Nazareth I, Petersen I. Trends in incidence, prevalence and prescribing in type 2 diabetes mellitus between 2000 and 2013 in primary care: a retrospective cohort study. BMJ Open 2016;6:e010210.

24 Overbeek JA, Heintjes EM, Prieto-Alhambra D, et al. Type 2 diabetes mellitus treatment patterns across Europe: a population-based multidatabase study. Clin Ther 2017;39:759-70.

25 Wang C, Gao Y, Zhu L, et al. Treatment patterns in patients with newly diagnosed type 2 diabetes in China: a retrospective, longitudinal database study. Clin Ther 2019;41:1440-52.

26 Ministry of Health. HISO 10001:2017 Ethnicity Data Protocols. Wellington: Ministry of Health, 2017. www.health.govt.nz/publication/ hiso-100012017-ethnicity-data-protocols

27 Atkinson J, Salmond C, Crampton P. NZDep2013 Index of Deprivation. Wellington: Department of Public Health, University of Otago, 2014. www.health.govt.nz/publication/nzdep2013-indexdeprivation 